\title{
A THEORY OF APPROXIMATE REASONING WITH TYPE-2 FUZZY SET
}

\author{
Sudin Manda ${ }^{1, a}$, InJamam Ul Karim ${ }^{1, b}$, And Swapan Raha ${ }^{1, c}$ \\ ${ }^{1}$ Department of Mathematics, Visva-Bharati, Santiniketan, West-Bengal, \\ India-731235, ${ }^{a}$ sudin.mandal@rediffmail.com, ${ }^{b}$ injymath@gmail.com, \\ ${ }^{c}$ swapan.raha@visva-bharati.ac.in
}

\begin{abstract}
In this paper, an attempt is made to study approximate reasoning based on a Type- 2 fuzzy set theory. In the process, we have examined the underlying fuzzy logic structure on which the reasoning is formulated. We have seen that the partial/incomplete/imprecise truth-values of elements of a type-2 fuzzy set under consideration forms a lattice. We propose two new lattice operations which ultimately help us to define a residual and thereby making the structure of truthvalues a residuated lattice. We have focused upon two typical rules of inference used mostly in ordinary approximate reasoning methodology based on Type-1 fuzzy set theory. The proposal is illustrated with typical artificial examples.

Key words and Phrases: Type-2 fuzzy set, Type-2 fuzzy logic, Generalised Modus Ponens, Approximate reasoning
\end{abstract}

\section{INTRODUCTION}

In 1965, the concept of a fuzzy set was introduced by Zadeh[16] and it has already established its usefulness through successful applications in different fields. Considering the importance of fuzzy logic as a basis for approximate reasoning, a systematic development of fuzzy set theory, the deductive aspects and structures of the underlying fuzzy logics were extensively studied[2]. In dealing with vagueness/impreciseness using fuzzy set theory we come across situations, where it is difficult to find satisfactorily the degree of membership of an element of the universal set in a particular fuzzy subset. This motivated Zadeh to introduce a generalization of fuzzy set, a Type-2 fuzzy set, in 1975[17]. The key point in this generalization is that membership degrees of elements in a Type-2 fuzzy set are the traditional fuzzy sets in $[0,1]$, while that for ordinary fuzzy sets are real numbers in $[0,1]$. Accordingly, any Type-2 fuzzy logic is a generalization of some fuzzy

2020 Mathematics Subject Classification: 03E72

Received: 06-02-2020, accepted: 12-02-2021. 
logic with Type-1 fuzzy set as proposed by Zadeh[2, 18]. The uncertainty in setting membership degree of an element to belong to a fuzzy set makes the theory of Type-2 fuzzy sets a convenient tool to handle higher degree of uncertainty in practice.

Approximate reasoning methodology with Type-2 fuzzy set is developed to formulate complex problems of human reasoning. The main motivation of the theory of approximate reasoning with Type- 2 fuzzy set is apparently, the desire to build up a qualitative framework that will allow one to derive an approximate conclusion from a collection of imprecise knowledge. Fuzzy logic is the basis of approximate reasoning with Type-2 fuzzy set. Fuzzy sets and fuzzy relations are used to represent, simple and complex fuzzy propositions in any Type- 2 fuzzy logic. Rules of inference are used to derive new propositions (fuzzy logical forms) from an observed data and given knowledge on the same.

Derivation of an imprecise statement from a set of imprecise statements is efficiently performed by humans. For a better understanding of how human beings assess knowledge in problem-solving, categorization, information retrieval and reasoning we have studied different aspects of fuzzy reasoning for an adequate theory of approximate reasoning with fuzzy sets of Type- 2 .

Based on the concept of fuzzy sets of Type 2 (or fuzzy-fuzzy sets) defined by L. A. Zadeh, authors in [6, 7, 8] studied fuzzy automata and investigated some of their properties. In a second research, the authors investigated the algebraic structures of fuzzy grades under the operations of join $\cup$, meet $\cap$, and negation $\sim$ which were defined by using the extension principle. Moreover, the algebraic properties of fuzzy grades under two new operations $\sqcup$ and $\sqcap$ which are slightly different from $\cup$ and $\cap$, respectively, were briefly discussed. In another article, the authors examined some algebraic properties of fuzzy grades (that is, fuzzy sets of Type 2) under the operations of algebraic product and algebraic sum which could be defined by using the concept of the extension principle and showed that fuzzy grades under these operations do not form such algebraic structures as a lattice and/or a semi-ring. Nilesh N. Karnik, Jerry M. Mendel and Qilian Liang [4] introduced a Type-2 fuzzy logic system (FLS), which can handle rule uncertainties. The implementation of this Type-2 FLS involves the operations of fuzzification, inference, and output processing which, consists of type reduction and defuzzification. They also applied a Type-2 FLS to time-varying channel equalization and demonstrated that it provides better performance than a Type-1 FLS and nearest neighbour classifier. In a research work[5], the authors established a small set of terms that allowed us communicate with Type-2 fuzzy sets and also helped us define such sets rather precisely. There they presented a new representation for Type-2 fuzzy sets, and used it to derive formulas for union, intersection and complement of Type-2 fuzzy sets without having the use of extension principle. C.L.Walker and E.A.Walker in [13, 14, 15], presented a straightforward mathematical treatment of algebras of fuzzy truth values for Type-2 fuzzy sets. They investigated automorphisms of the algebra of truth values of Type- 2 fuzzy sets. This algebra contains isomorphic copies of the truth value algebras of Type-1 and of interval-valued fuzzy 
sets. It is shown that these subalgebras are characteristic; that is, are carried onto themselves by automorphisms of the containing algebra of truth values of fuzzy sets. The algebra of truth values of type-2 fuzzy sets consists of all mappings of the unit interval to itself, with Type- 2 operations that are convolutions of ordinary max and min operations. Their work was concerned with a special subalgebra of this truth value algebra, namely, the set of non-zero functions with values in the two-element set $\{0,1\}$. This algebra can be identified with the set of all non-empty subsets of the unit-interval, but the operations are not the usual union and intersection.

This research work is organized into seven sections. In section 2 , we define a few terms in order to communicate with Type-2 fuzzy sets. Aspects of reasoning are considered in the context of formation of a Type- 2 fuzzy set theory. In section 3 , we have examined the existence of a lattice structure for the truth-values of elements in a Type- 2 fuzzy set. Section 4 of this paper examines basic definitions and their use in the context of defining a methodology of reasoning with Type- 2 fuzzy set. In section 5, a computational procedure is presented to demonstrate the computations involved in reasoning with Type-2 fuzzy sets. Two basic rules of inferences, CRI and GMP, have been developed/formulated. Examples are considered to illustrate the problem. The work is briefly concluded in section 6 . This is followed by a list of references in the last section.

\section{MATHEMATICAL PRELIMINARIES}

In this research, we attempt to study reasoning under Type-2 fuzzy logic. Accordingly, a brief study on the theory of Type-2 fuzzy sets is presented at first. We focus, in particular, on the study of operations on Type- 2 fuzzy sets. The concept of a Type-2 fuzzy relation and fuzzy connectives not $(\neg)$, and $(\wedge)$ and or $(\vee)$ are also studied. Appropriate interpretation of connectives is one of the basic problems in any fuzzy logic and its application. Classes of negation functions (to model complement operators), continuous triangular norms (to model conjunction) and triangular co-norms ( to model disjunction) are also been examined extensively $[13,14,15]$. These classes of operations are found to be mathematically sound and contain a wide variety of particular members.

Let $F$ be the set of all fuzzy subsets over $[0,1]$. In order to make $F$ a poset, we define the relation ' $\leq$ ' as ' $A \leq B$ ' if and only if $(\forall j)_{[0,1]} A(j) \leq B(j)$. Defining $\mathbb{1}(j)=1$ and $0(j)=0(\forall j)_{[0,1]}$, we propose $F$ to be a bounded class. Now, the t-norm, t-conorm and implication for a Type- 2 fuzzy logical setting would be characterised as in the following:

Definition 2.1. A binary operation $T: F \times F \rightarrow F$ is called a Type-2 $t$-norm if for every $A_{1}, A_{2}, A_{3}, A_{4} \in F$.
i) $T\left(\mathbb{1}, A_{1}\right)=A_{1}$,
ii) $T\left(A_{1}, A_{2}\right)=T\left(A_{2}, A_{1}\right)$,
iii) $T\left(A_{1}, T\left(A_{2}, A_{3}\right)\right)=T\left(T\left(A_{1}, A_{2}\right), A_{3}\right)$,
iv) if $A_{1} \leq A_{2}$ and $A_{3} \leq A_{4}$ then $T\left(A_{1}, A_{3}\right) \leq T\left(A_{2}, A_{4}\right)$. 
We note that condition i) implies $T(\mathbb{1}, \mathbb{1})=\mathbb{1}$, condition i) and ii) imply $T(\mathbb{1}, 0)=T(0, \mathbb{1})=0$ and from condition i) and iv) we get $T(0,0)=0$, which are the basic properties for crisp intersection.

Example 2.2. Define $T_{1}, T_{2}: F \times F \rightarrow F$ by

$$
T_{1}\left(A_{1}, A_{2}\right)=\sum_{j \in[0,1]} \frac{\min \left(A_{1}(j), A_{2}(j)\right)}{j}
$$

and

$$
T_{2}\left(A_{1}, A_{2}\right)=\sum_{j \in[0,1]} \frac{\max \left(A_{1}(j)+A_{2}(j)-1,0\right)}{j}
$$

where,

$$
A_{1}=\sum_{j \in[0,1]} \frac{A_{1}(j)}{j} ; \quad A_{2}=\sum_{j \in[0,1]} \frac{A_{2}(j)}{j} .
$$

It is easily noticeable that $T_{1}$ and $T_{2}$ are Type-2 t-norms. We note here that $T_{1}\left(A_{1}, A_{1}\right)=A_{1}$ and $T_{2}\left(A_{1}, A_{1}\right) \neq A_{1}$ i.e., $T_{1}$ is idempotent but $T_{2}$ is not. Again, we note that $T_{1}\left(A_{1}, A_{1}\right) \nless A_{1}$ but $T_{2}\left(A_{1}, A_{1}\right)<A_{1}$ this means $T_{2}$ is Archimedean while $T_{1}$ is not.

Definition 2.3. A binary operation $S: F \times F \rightarrow F$ is called a Type-2 t-conorm if for all $A_{1}, A_{2}, A_{3}, A_{4} \in F$.

i) $S\left(0, A_{1}\right)=A_{1}$,

ii) $S\left(A_{1}, A_{2}\right)=S\left(A_{2}, A_{1}\right)$,

iii) $S\left(A_{1}, S\left(A_{2}, A_{3}\right)\right)=S\left(S\left(A_{1}, A_{2}\right), A_{3}\right)$,

iv) if $A_{1} \leq A_{2}$ and $A_{3} \leq A_{4}$ then $S\left(A_{1}, A_{3}\right) \leq S\left(A_{2}, A_{4}\right)$.

Similar to t-norm, we see that

$$
S(\mathbb{1}, \mathbb{1})=S(0, \mathbb{1})=S(\mathbb{1}, 0)=\mathbb{1} \text { and } S(0,0)=0 .
$$

Example 2.4. Define $S_{1}, S_{2}: F \times F \rightarrow F$ by

$$
S_{1}\left(A_{1}, A_{2}\right)=\sum_{j \in[0,1]} \frac{\max \left(A_{1}(j), A_{2}(j)\right)}{j}
$$

and

$$
S_{2}\left(A_{1}, A_{2}\right)=\sum_{j \in[0,1]} \frac{\min \left(A_{1}(j)+A_{2}(j), 1\right)}{j} .
$$

Here, $S_{1}$ and $S_{2}$ are both examples of Type-2 t-conorm. Also, $S_{1}$ is idempotent but not Archimedean whereas $S_{2}$ is Archimedean but not idempotent.

Definition 2.5. A mapping $N: F \rightarrow F$ is called a Type-2 negation operator which satisfies
i) if $A_{1} \geq A_{2}$ then $N\left(A_{1}\right) \leq N\left(A_{2}\right)$,
ii) $N\left(N\left(A_{1}\right)\right)=A_{1}$,
iii) $N(\mathbb{1})=0$,
iv) $N(0)=\mathbb{1}$ 
Example 2.6. For example $N: F \rightarrow F$ defined by $N\left(A_{1}\right)=\mathbb{1}-A_{1}$, where' ${ }^{\prime}{ }^{\text {is }}$ taken argumentwise, is an obvious choice for negation.

Definition 2.7. The binary operations $\vee, \wedge$ on $F$ are defined

$$
\begin{gathered}
\left(\forall A_{1}, A_{2}\right)_{F} A_{1} \vee A_{2}=S\left(A_{1}, A_{2}\right) \\
\text { and } A_{1} \wedge A_{2}=T\left(A_{1}, A_{2}\right) .
\end{gathered}
$$

Some other important properties for t-norm, t-conorm and negation are stated as in the following

(A) Absorption law:

i) $S\left(A_{1}, T\left(A_{1}, A_{2}\right)\right)=A_{1}$,

ii) $T\left(A_{1}, S\left(A_{1}, A_{2}\right)\right)=A_{1}$;

(B) Distributive law:

i) $T\left(S\left(A_{1}, A_{2}\right), S\left(A_{1}, A_{3}\right)\right)=S\left(A_{1}, T\left(A_{2}, A_{3}\right)\right)$,

ii) $S\left(T\left(A_{1}, A_{2}\right), T\left(A_{1}, A_{3}\right)\right)=T\left(A_{1}, S\left(A_{2}, A_{3}\right)\right)$;

(C) De Morgan's law:

i) $N\left(T\left(A_{1}, A_{2}\right)\right)=S\left(N\left(A_{1}\right), N\left(A_{2}\right)\right)$,

ii) $N\left(S\left(A_{1}, A_{2}\right)\right)=T\left(N\left(A_{1}\right), N\left(A_{2}\right)\right)$;

(D) Complementation law:

i) $S\left(A_{1}, N\left(A_{1}\right)\right)=\mathbb{1}$,

ii) $T\left(A_{1}, N\left(A_{1}\right)\right)=0$.

It can be shown that $T_{1}$ along with $S_{1}$ and $N$ satisfies absorption, distributive and De Morgan's laws but not complementation law, also $T_{2}$ along with $S_{2}$ and $N$ satisfies De Morgan's law and complementation law but neither absorption nor distributive laws.

Definition 2.8. For every $A_{1}, A_{2}, A_{3} \in F$; a binary operation $I: F \times F \rightarrow F$ is called a Type-2 implication which satisfies

I1) if $A_{1} \leq A_{2}$ then $I\left(A_{3}, A_{2}\right) \geq I\left(A_{3}, A_{1}\right)$,

I2) if $A_{1} \leq A_{2}$ then $I\left(A_{1}, A_{3}\right) \geq I\left(A_{2}, A_{3}\right)$,

I3) $I\left(0, A_{2}\right)=\mathbb{1}$,

I4) $I\left(1, A_{2}\right)=A_{2}$

It can be seen that $I(\mathbb{1}, \mathbb{1})=I(0,0)=I(0, \mathbb{1})=\mathbb{1}$ and $I(\mathbb{1}, 0)=0$.

Example 2.9. Define $I_{1}, I_{2}: F \times F \rightarrow F$ by

$$
I_{1}\left(A_{1}, A_{2}\right)=\sum_{j \in[0,1]} \frac{\max \left(1-A_{1}(j), A_{2}(j)\right)}{j}
$$

and

$$
I_{2}\left(A_{1}, A_{2}\right)=\sum_{j \in[0,1]} \frac{\min \left(1-A_{1}(j)+A_{2}(j), 1\right)}{j} .
$$

It is easy to see that $I_{1}$ and $I_{2}$ are examples of two Type-2 implicators.

Some other important properties for implication operators are 
I5) $I\left(A_{1}, A_{2}\right) \geq A_{2}$;

I6) $I\left(A_{1}, A_{1}\right)=\mathbb{1}$;

I7) $I\left(A_{1}, I\left(A_{2}, A_{3}\right)=I\left(A_{2}, I\left(A_{1}, A_{3}\right)\right.\right.$;

I8) $I\left(A_{1}, A_{2}\right)=\mathbb{1}$ if and only if $A_{1} \leq A_{2}$ and;

I9) $I\left(A_{1}, A_{2}\right)=I\left(N\left(A_{2}\right), N\left(A_{1}\right)\right)$ for some negation operator $N$.

Implication operator, $I_{1}$ satisfies I5,I7,I9 and $I_{2}$ satisfies all of them.

\section{Formation of Lattice}

First we note that $F$ together with $\wedge, \vee, \mathbb{1}$ and 0 defined as above, forms a complete, distributive lattice. We define $*: F \times F \rightarrow F$ by

$$
*\left(A_{1}, A_{2}\right)=A_{1} * A_{2}=\sum_{j \in[0,1]} \frac{\max \left(A_{1}(j)+A_{2}(j)-1,0\right)}{j} .
$$

It is easy to see that $(F, *)$ forms a commutative monoid [1,9] . Now as Pavelka[10, $11,12]$ said there must be an unique residual $\rightarrow$ given by

$$
A_{1} \rightarrow A_{2}=\vee\left\{X: X * A_{1} \leq A_{2}\right\} .
$$

As for any $X \in F$

$$
\begin{array}{rlc}
(\forall j)_{[0,1]}, \quad & & \max \left(A_{1}(j)+X(j)-1,0\right) \leq A_{2}(j) \\
& \Leftrightarrow \quad X(j) \leq 1-A_{1}(j)+A_{2}(j) \\
& \Leftrightarrow \quad X(j) \leq \min \left(1,1-A_{1}(j)+A_{2}(j)\right) .
\end{array}
$$

Obviously, the equality occurs for $X=\mathbb{1}$, which means

$$
A_{1} \rightarrow A_{2}=\vee\left\{X: X * A_{1} \leq A_{2}\right\}=\min \left(1,1-A_{1}(j)+A_{2}(j)\right)
$$

Now we have a complete residuated lattice $\langle F, \vee, \wedge, *, \rightarrow\rangle$ of truth values to develop a Type-2 logic.

Now, if we set $A=\sum_{x \in U} \frac{A_{x}=\sum_{j \in[0,1]} \frac{A_{x}(j)}{j}}{x}$ and $B=\sum_{y \in V} \frac{B_{y}=\sum_{j \in[0,1]} \frac{B_{y}(j)}{j}}{y}$ then we have $A_{x} *\left(A_{x} \rightarrow B_{y}\right)$

$$
\begin{aligned}
& =\sum_{j} \frac{\max \left[\min \left(1-A_{x}(j)+B_{y}(j), 1\right)+A_{x}(j)-1,0\right]}{j} \\
& =\sum_{j} \frac{\max \left[A_{x}(j)-\max \left(A_{x}(j)-B_{y}(j), 0\right), 0\right]}{j} \\
& =\sum_{j} \frac{\min \left(A_{x}(j), B_{y}(j)\right.}{j} \leq B_{y} \text { (Fuzzy form of modus ponens). }
\end{aligned}
$$


Similarly,

$$
B_{y}^{c} *\left(A_{x} \rightarrow B_{y}\right)=\sum_{j} \frac{\min \left(A_{x}^{c}(j), B_{y}^{c}(j)\right.}{j} \leq A_{x}^{c} \text { (Fuzzy form of modus tollens). }
$$

where $A_{x}^{c}$ and $B_{y}^{c}$ respectively denote the fuzzy compement of $A_{x}$ and $B_{y}$. A detailed discussion of the properties of $*$ and $\rightarrow$ can be found in $[10,11,12]$.

\section{Logical OPERATIONS USING TyPe-2 FUZZY SET}

In this section, we will examine the formation of a typical Type-2 fuzzy logic with the help of the lattice of truth values as proposed in the previous section. The procedure is the same as in the case of a logic with Type-1 (ordinary) fuzzy set. Here, instead of a number from the unit interval we have to consider a Type-1 fuzzy subset over $[0,1]$. Their composition have already been defined pointwise. We have seen that the class of all linguistic truth values $\langle F, \wedge, \vee, 0, \mathbb{1}\rangle$ forms a complete, distributive lattice like the unit interval under the usual operations max, min. Accordingly, we are to state explicitly how reasoning can be made with typical knowledge expressed using Type-2 fuzzy sets and fuzzy relations.

Definition 4.1. Let $U=\left\{x_{1}, x_{2}, \cdots, x_{n}\right\}$ be a finite universal set. A Type-2 fuzzy set $A$ over the universe of discourse $U$ is characterized by a fuzzy membership function i.e., an object having the form

$$
A=\sum_{x \in U} \frac{A(x)=\sum_{j \in[0,1]} \frac{A_{x}(j)}{j}}{x} .
$$

Example 4.2. Let $A=\left\{\frac{A_{u_{1}}}{u_{1}}+\frac{A_{u_{2}}}{u_{2}}+\frac{A_{u_{3}}}{u_{3}}\right\}$ and $B=\left\{\frac{B_{v_{1}}}{v_{1}}+\frac{B_{v_{2}}}{v_{2}}+\frac{B_{v_{3}}}{v_{3}}\right\}$ be two Type-2 fuzzy sets defined on the universe $U=\left\{u_{1}, u_{2}, u_{3}\right\}$ and $V=\left\{v_{1}, v_{2}, v_{3}\right\}$ respectively, where the fuzzy grades of $A, B$ at $u_{1}, u_{2}, u_{3}$ and $v_{1}, v_{2}, v_{3}$ are given as in the following:

$$
\begin{aligned}
& A_{u_{1}}=\frac{1}{0}+\frac{.8}{.2}+\frac{.5}{.4}+\frac{.4}{.6}+\frac{.1}{.8}+\frac{0}{1}, \\
& A_{u_{2}}=\frac{.4}{0}+\frac{.8}{.2}+\frac{1}{.4}+\frac{.8}{.6}+\frac{.5}{.8}+\frac{.2}{1} \\
& A_{u_{3}}=\frac{0}{0}+\frac{0}{.2}+\frac{.2}{.4}+\frac{.5}{.6}+\frac{.7}{.8}+\frac{.9}{1} \\
& B_{v_{1}}=\frac{0}{0}+\frac{0}{.2}+\frac{.2}{.4}+\frac{.5}{.6}+\frac{.7}{.8}+\frac{.9}{1} \\
& B_{v_{2}}=\frac{1}{0}+\frac{.8}{.2}+\frac{.5}{.4}+\frac{.4}{.6}+\frac{.1}{.8}+\frac{0}{1} \\
& B_{v_{3}}=\frac{.4}{0}+\frac{.8}{.2}+\frac{1}{.4}+\frac{.8}{.6}+\frac{.5}{.8}+\frac{.2}{1} .
\end{aligned}
$$


Example 4.3. Let us consider $U=\{$ Chicoo, Litchie, Mango, Plum, Strawberry $\}$ to be a set of fruits and that $A$ is a fuzzy subset of type-2 of delicious fruit defined over $U$. We then express the same in set theoretic notation as,

$$
\begin{gathered}
A=\text { delicious fruit }=\frac{\text { not so delicious }}{\text { Chicoo }}+\frac{\text { fairly delicious }}{\text { Litchie }} \\
+\frac{\text { moderately delicious }}{\text { Plum }}+\frac{\text { very delicious }}{\text { Strawberry }}+\frac{\text { highly delicious }}{\text { Mango }},
\end{gathered}
$$

where, the fuzzy grades labelled moderately, fairly, highly are assumed to be fuzzy sets in

$J=0,0.1, \cdots, 0.9,1 \subseteq[0,1]$ and, for example, are expressed as in the following:

$$
\begin{gathered}
\text { moderately delicious }=\frac{0.5}{0.3}+\frac{0.75}{0.4}+\frac{1}{0.5}+\frac{0.75}{0.6}+\frac{0.5}{0.7} \\
\text { fairly delicious }=\frac{1}{0.0}+\frac{0.75}{0.1}+\frac{0.5}{0.2}+\frac{0.25}{0.3} \\
\text { highly delicious }
\end{gathered}
$$

Moreover, fuzzy grades for elements that belong to fuzzy sets such as not so delicious and very delicious are determined from fuzzy grades of the same as in delicious by using the concept of linguistic hedges as in modification of ordinary fuzzy sets. Thus,

$$
\begin{gathered}
\text { delicious }=\frac{0.25}{(0.3,0.9)}+\frac{0.5}{(0.4,0.8)}+\frac{0.75}{(0.5,0.7)}+\frac{1.0}{0.6} \\
\text { not so delicious }=\frac{1.0}{(0.1,0.2,1.0)}+\frac{0.75}{(0.3,0.9)}+\frac{0.5}{(0.4,0.8)}+\frac{0.25}{(0.5,0.7)} \\
\text { very delicious }=\frac{0.0625}{(0.3,0.9)}+\frac{0.25}{(0.4,0.8)}+\frac{0.5625}{(0.5,0.7)}+\frac{1.0}{0.6}
\end{gathered}
$$

Let $A$ and $B$ be two fuzzy sets of Type-2 over the universe of discourse $U$ and $A_{x}(j)$ and $B_{x}(j)$ be the fuzzy grades of the point $x$ in $A$ and $B$ respectively (that is, fuzzy subsets of $[0,1])$.

At each value of $x$, the plane section determined by $x$ and $A_{x}(j)$ for different values of $j$ is the vertical slice of $A$ at $x$. Vertical slices represent secondary membership of $x$ in $A$; it is a Type-1 fuzzy set over [0,1][3]. The domain of secondary membership function is regarded as the primary membership of $x$.

Now, for the operations on fuzzy sets of Type-2, let's choose a logic system and explore its several properties. 
Definition 4.4. The union of two Type-2 fuzzy sets $A$ and $B$ defined over $X$ is actually a logical disjunction between the truth values $A_{x}$ and $B_{x}$ for every $x \in X$, denoted by $A \sqcup B$ and is defined by

$$
A \sqcup B=\sum_{x \in X} \frac{A_{x} \vee B_{x}}{x} .
$$

where $\vee$ is an operation on the elements the class $F$.

Definition 4.5. The intersection of two Type-2 fuzzy sets $A$ and $B$ defined over $X$ is actually a logical conjunction between the truth values $A_{x}$ and $B_{x}$ for every $x \in X$, denoted by $A \sqcap B$ and is defined by

$$
A \sqcap B=\sum_{x \in X} \frac{A_{x} \wedge B_{x}}{x} .
$$

where $\wedge$ is interpreted as before.

Definition 4.6. The complement of a Type-2 fuzzy set A defined over $X$, denoted by $A^{c}$ and is defined by

$$
A^{c}=\sum_{x \in X} \frac{\mathbb{1}-A_{x}}{x} .
$$

Definition 4.7. Let $A$ and $B$ be two Type-2 fuzzy sets over $X, Y$ and whose membership grade values at $x \in X$ and $y \in Y$ are $A_{x}$ and $B_{y}$ respectively. Then by the conditional statement ' $A$ implies $B$ ', we mean a Type-2 fuzzy relation between $X$ and $Y$ whose membership grade at a point $(x, y) \in X \times Y$ is $A_{x} \rightarrow B_{y}$ where, $\rightarrow$ is from the lattice $F$.

Thus,

where $\rightarrow$ is taken from the lattice $F$.

$$
A \Longrightarrow B=\sum_{(x, y) \in X \times Y} \frac{\left(A_{x} \rightarrow B_{y}\right)}{(x, y)} .
$$

Example 4.8. Let $J=\{0,0.1, \cdots, 0.9,1.0\}$ and let fuzzy grades $A_{x}$ and $B_{x}$ be given as

Then, we have

$$
\begin{aligned}
& A_{x}=\frac{0.5}{0.0}+\frac{0.7}{0.1}+\frac{0.3}{0.2} \\
& B_{x}=\frac{0.9}{0.0}+\frac{0.6}{0.1}+\frac{0.2}{0.2}
\end{aligned}
$$

$$
(A \sqcup B)_{x}(j)=\max \left\{A_{x}(j), B_{x}(j)\right\}=\frac{0.9}{0.0}+\frac{0.7}{0.1}+\frac{0.3}{0.2} .
$$

Similarly, we have

and

$$
(A \sqcap B)_{x}(j)=\min \left\{A_{x}(j), B_{x}(j)\right\}=\frac{0.5}{0.0}+\frac{0.6}{0.1}+\frac{0.2}{0.2} ;
$$

$$
A_{x}^{c}(j)=\left\{1-A_{x}(j)\right\}=\frac{0.5}{0.0}+\frac{0.3}{0.1}+\frac{0.7}{0.2}+\frac{1.0}{(0.3,0.4, \cdots 1.0)}
$$


Example 4.9. The following table describes the relation $A \Longrightarrow B$ taking $A$ and $B$ from Example 5.

TABle 1 . The Type-2 fuzzy relation $A \Longrightarrow B$

\begin{tabular}{|c|c|c|c|}
\hline & $v_{1}$ & $v_{2}$ & $v_{3}$ \\
\hline$u_{1}$ & $\frac{0.0}{0.0}+\frac{0.2}{0.2}+\frac{0.7}{0.4}+\frac{1.0}{0.6}+\frac{1.0}{0.8}+\frac{1.0}{1.0}$ & $\frac{1.0}{0.0}+\frac{1.0}{0.2}+\frac{1.0}{0.4}+\frac{1.0}{0.6}+\frac{1.0}{0.8}+\frac{1.0}{1.0}$ & $\frac{0.4}{0.0}+\frac{1.0}{0.2}+\frac{1.0}{0.4}+\frac{1.0}{0.6}+\frac{1.0}{0.8}+\frac{1.0}{1.0}$ \\
\hline$u_{2}$ & $\frac{0.6}{0.0}+\frac{0.2}{0.2}+\frac{0.2}{0.4}+\frac{0.7}{0.6}+\frac{1.0}{0.8}+\frac{1.0}{1.0}$ & $\frac{1.0}{0.0}+\frac{1.0}{0.2}+\frac{0.5}{0.4}+\frac{0.6}{0.6}+\frac{0.6}{0.8}+\frac{0.8}{1.0}$ & $\frac{1.0}{0.0}+\frac{1.0}{0.2}+\frac{1.0}{0.4}+\frac{1.0}{0.6}+\frac{1.0}{0.8}+\frac{1.0}{1.0}$ \\
\hline$u_{3}$ & $\frac{1.0}{0.0}+\frac{1.0}{0.2}+\frac{1.0}{0.4}+\frac{1.0}{0.6}+\frac{1.0}{0.8}+\frac{1.0}{1.0}$ & $\frac{1.0}{0.0}+\frac{1.0}{0.2}+\frac{1.0}{0.4}+\frac{0.9}{0.6}+\frac{0.4}{0.8}+\frac{0.1}{1.0}$ & $\frac{1.0}{0.0}+\frac{1.0}{0.2}+\frac{1.0}{0.4}+\frac{1.0}{0.6}+\frac{0.8}{0.8}+\frac{0.3}{1.0}$ \\
\hline
\end{tabular}

Definition 4.10. Let $R$ be a Type-2 fuzzy relation defined on $U \times V$, and is explicitly given by

$$
R(x, y)=\sum_{j \in[0,1]} \frac{R_{(x, y)}(j)}{j} .
$$

The projection of $R$ on $U$ is a Type-2 fuzzy set $A$ defined over $U$ and is explicitly given by

$$
A_{x}(j)=\max _{y \in V}\left\{R_{(x, y)}(j)\right\}
$$

Example 4.11. Let $R$ be a Type-2 fuzzy relation over $U \times V$ and let it be given by the following table

TABLE 2. An artificial Type-2 fuzzy relation $\mathrm{R}$

\begin{tabular}{|l|c|c|c|}
\hline & $v_{1}$ & $v_{2}$ & $v_{3}$ \\
\hline$u_{1}$ & $\frac{0.0}{0.0}+\frac{0.2}{0.2}+\frac{0.5}{0.4}+\frac{0.6}{0.6}+\frac{0.9}{0.8}+\frac{1.0}{1.0}$ & $\frac{1.0}{0.0}+\frac{0.8}{0.2}+\frac{0.5}{0.4}+\frac{0.6}{0.6}+\frac{0.9}{0.8}+\frac{1.0}{1.0}$ & $\frac{0.6}{0.0}+\frac{0.8}{0.2}+\frac{1.0}{0.4}+\frac{0.8}{0.6}+\frac{0.5}{0.8}+\frac{0.8}{1.0}$ \\
\hline$u_{2}$ & $\frac{0.6}{0.0}+\frac{0.2}{0.2}+\frac{0.2}{0.4}+\frac{0.5}{0.6}+\frac{0.7}{0.8}+\frac{0.9}{1.0}$ & $\frac{1.0}{0.0}+\frac{0.8}{0.2}+\frac{0.5}{0.4}+\frac{0.4}{0.6}+\frac{0.5}{0.8}+\frac{0.8}{1.0}$ & $\frac{0.6}{0.0}+\frac{0.8}{0.2}+\frac{0.1}{0.4}+\frac{0.8}{0.6}+\frac{0.5}{0.8}+\frac{0.8}{1.0}$ \\
\hline$u_{3}$ & $\frac{1.0}{0.0}+\frac{1.0}{0.2}+\frac{0.8}{0.4}+\frac{0.5}{0.6}+\frac{0.7}{0.8}+\frac{0.9}{1.0}$ & $\frac{1.0}{0.0}+\frac{1.0}{0.2}+\frac{0.8}{0.4}+\frac{0.5}{0.6}+\frac{0.3}{0.8}+\frac{0.1}{1.0}$ & $\frac{1.0}{0.0}+\frac{1.0}{0.2}+\frac{1.0}{0.4}+\frac{0.8}{0.6}+\frac{0.5}{0.8}+\frac{0.2}{1.0}$ \\
\hline
\end{tabular}


Then, the projection of $R$ over $V$ is a Type-2 fuzzy set $P$ where

$$
\begin{aligned}
& P\left(v_{1}\right)=\max \left(R\left(u_{1}, v_{1}\right), R\left(u_{2}, v_{1}\right), R\left(u_{3}, v_{1}\right)\right)=\frac{1.0}{0.0}+\frac{1.0}{0.2}+\frac{0.8}{0.4}+\frac{0.6}{0.6}+\frac{0.9}{0.8}+\frac{1.0}{1.0}, \\
& P\left(v_{2}\right)=\frac{1.0}{0.0}+\frac{1.0}{0.2}+\frac{0.8}{0.4}+\frac{0.6}{0.6}+\frac{0.9}{0.8}+\frac{1.0}{1.0} \\
& \text { and } P\left(v_{3}\right)=\frac{1.0}{0.0}+\frac{1.0}{0.2}+\frac{1.0}{0.4}+\frac{0.8}{0.6}+\frac{0.5}{0.8}+\frac{0.8}{1.0} .
\end{aligned}
$$

Definition 4.12. Let $U, V$ be two universes of discourses, and $A=\sum_{x \in U} \frac{A_{x}}{x}$ be a Type-2 fuzzy set defined over $U$. The cylindrical extension of $A$ to $U \times V$ is a Type-2 fuzzy relation $\widetilde{c e(A)}$ defined by

$$
\widetilde{c e(A)}=\sum_{(x, y) \in U \times V} \frac{\widetilde{c e(A)}(x, y)}{(x, y)} \text {, where } x \in U, y \in V
$$

and

$$
\widetilde{c e(A)}_{(x, y)}(j)=A_{x}(j) .
$$

Thus, the cylindrical extension clearly produces the largest fuzzy relation that is compatible with the given projection. Such a relation is the least specific of all relations compatible with the projection.

Example 4.13. Let $A_{u_{1}}, A_{u_{2}}, A_{u_{3}}$ be the elements of a Type-2 fuzzy set over the universe $U$ where,

and

$$
\begin{aligned}
& A_{u_{1}}=\frac{1.0}{0.0}+\frac{0.8}{0.2}+\frac{0.5}{0.4}+\frac{0.4}{0.6}+\frac{0.1}{0.8}+\frac{0.0}{1.0} \\
& A_{u_{2}}=\frac{0.4}{0.0}+\frac{0.8}{0.2}+\frac{1.0}{0.4}+\frac{0.8}{0.6}+\frac{0.5}{0.8}+\frac{0.2}{1.0}, \\
& A_{u_{3}}=\frac{0.0}{0.0}+\frac{0.0}{0.2}+\frac{0.2}{0.4}+\frac{0.5}{0.6}+\frac{0.7}{0.8}+\frac{0.9}{1.0},
\end{aligned}
$$

$$
A=\sum_{i=1}^{3} \frac{A_{u_{i}}}{u_{i}}
$$

Then, its cylindrical extension over $U \times V$ where, $V=\left\{v_{1}, v_{2}, v_{3}\right\}$, is given by

$$
\begin{aligned}
& \tilde{A}\left(u_{1}, v_{1}\right)=A_{u_{1}}=\frac{1.0}{0.0}+\frac{0.8}{0.2}+\frac{0.5}{0.4}+\frac{0.4}{0.6}+\frac{0.1}{0.8}+\frac{0.0}{1.0}, \\
& \tilde{A}\left(u_{2}, v_{1}\right)=A_{u_{2}}=\frac{0.4}{0.0}+\frac{0.8}{0.2}+\frac{1.0}{0.4}+\frac{0.8}{0.6}+\frac{0.5}{0.8}+\frac{0.2}{1.0}, \\
& \tilde{A}\left(u_{3}, v_{1}\right)=A_{u_{3}}=\frac{0.0}{0.0}+\frac{0.0}{0.2}+\frac{0.2}{0.4}+\frac{0.5}{0.6}+\frac{0.7}{0.8}+\frac{0.9}{1.0}, \\
& \tilde{A}\left(u_{1}, v_{2}\right)=A_{u_{1}}=\frac{1.0}{0.0}+\frac{0.8}{0.2}+\frac{0.5}{0.4}+\frac{0.4}{0.6}+\frac{0.1}{0.8}+\frac{0.0}{1.0},
\end{aligned}
$$




$$
\begin{aligned}
& \tilde{A}\left(u_{2}, v_{2}\right)=A_{u_{2}}=\frac{0.4}{0.0}+\frac{0.8}{0.2}+\frac{1.0}{0.4}+\frac{0.8}{0.6}+\frac{0.5}{0.8}+\frac{0.2}{1.0}, \\
& \tilde{A}\left(u_{3}, v_{2}\right)=A_{u_{3}}=\frac{0.0}{0.0}+\frac{0.0}{0.2}+\frac{0.2}{0.4}+\frac{0.5}{0.6}+\frac{0.7}{0.8}+\frac{0.9}{1.0}, \\
& \tilde{A}\left(u_{1}, v_{3}\right)=A_{u_{1}}=\frac{1.0}{0.0}+\frac{0.8}{0.2}+\frac{0.5}{0.4}+\frac{0.4}{0.6}+\frac{0.1}{0.8}+\frac{0.0}{1.0}, \\
& \tilde{A}\left(u_{2}, v_{3}\right)=A_{u_{2}}=\frac{0.4}{0.0}+\frac{0.8}{0.2}+\frac{1.0}{0.4}+\frac{0.8}{0.6}+\frac{0.5}{0.8}+\frac{0.2}{1.0}, \\
& \tilde{A}\left(u_{3}, v_{3}\right)=A_{u_{3}}=\frac{0.0}{0.0}+\frac{0.0}{0.2}+\frac{0.2}{0.4}+\frac{0.5}{0.6}+\frac{0.7}{0.8}+\frac{0.9}{1.0} .
\end{aligned}
$$

\section{Approximate Reasoning}

In this section, we demonstrate how conclusions can be obtained from given premises with the help of Type-2 fuzzy set theory. For that let $A$ and $B$ be two fuzzy sets of Type-2 over the universe of discourse $U$ and $V$ respectively. We consider the following interpretation of the logical connectives.

$A$ or $B$ translates to $\sum_{(x, y) \in U \times V} \frac{S(A(x), B(y))}{(x, y)} ; \mathrm{S}$ is a t-conorm,

$A$ and $B$ translates to $\sum_{(x, y) \in U \times V} \frac{T(A(x), B(y))}{(x, y)} ; \mathrm{T}$ is a t-norm,

A implies $B$ translates to $\sum_{(x, y) \in U \times V} \frac{I(A(x), B(y))}{(x, y)}$; I is a fuzzy implication

and not $A$ translates to $\sum_{x \in U} \frac{1-A(x)}{x}$.

According to Zadeh, approximate reasoning using the scheme

from ' $\mathrm{X}$ is $\mathrm{A}$ ' and ' $(\mathrm{X}, \mathrm{Y})$ is $\mathrm{R}$ ' infer ' $\mathrm{Y}$ is $\mathrm{B}$ '

is termed as the compositional rule of inference(as given in Table3).

TABle 3. Compositional Rule of Inference

$$
\begin{array}{ccc}
\mathrm{p}: & \mathrm{X} \text { is } A & \\
\mathrm{q}: & (\mathrm{X}, \mathrm{Y}) \text { is } R & \\
\hline \mathrm{r}: & & \mathrm{Y} \text { is } B .
\end{array}
$$

Here, $A$ is a type-2 fuzzy subset of $U, R$ is a type-2 fuzzy subset of $U \times V$ and $B$ is a fuzzy subset of $V$. We now present computations involved in the above 
inference mechanism in the following algorithm.

ALGORITHM OAR : Ordinary Approximate Reasoning based on CRI Input : $A$ and $R$

Output : $B$

Step 1. Represent $A$ as a Type-2 fuzzy subset over $U$ and $R$ as a Type-2 fuzzy relation over the universe of discourse $U \times V$.

Step 2. Compute $\widetilde{c e(A)}$, the cylindrical extension of $A$ over $U \times V$.

Step 3. Compose $\widehat{\operatorname{ce}(A)}$ and $R$ to form $S$, a fuzzy relation of Type-2 using some conjunction operation meant for fuzzy sets of Type-2.

Step 4. Compute $B=\operatorname{Proj}_{V} S$, i.e., $B=\sum_{y \in V} \frac{B(y)}{y}$ where,

$$
B(y)=\sum_{j \in[0,1]} \frac{\max _{x \in U} S_{j}(x, y)}{j} .
$$

Example 5.1. Let us consider $U=\{$ Aditi, Bidisha, Camellia $\}$ to be a set of women and that $A$ be a fuzzy subset of Type-2 of incredible women in $U$. Then, we may have

$$
\begin{gathered}
A=\frac{\text { more or less }}{\text { Aditi }}+\frac{\text { highly }}{\text { Bidisha }}+\frac{\text { not at all }}{\text { Camellia }} \\
=\frac{\frac{0.6}{0.4}+\frac{1}{0.5}+\frac{0.5}{0.6}+\frac{0.2}{0.7}}{\text { Aditi }}+\frac{\frac{0.3}{0.7}+\frac{0.6}{0.8}+\frac{0.8}{0.9}+\frac{1}{1}}{\text { Bidisha }}+\frac{\frac{1}{0.1}+\frac{0.8}{0.2}+\frac{0.5}{0.3}+\frac{0.2}{0.4}}{\text { Camellia }} .
\end{gathered}
$$

Let $V=\{$ Priti, Rina, Sarmishtha $\}$ be another set of women and that $B$ be the fuzzy set of Type-2 of smart woman defined on $V$.

Then,

$$
\begin{gathered}
B=\frac{\text { highly }}{\text { Priti }}+\frac{\text { more or less }}{\text { Rina }}+\frac{\text { not at all }}{\text { Sarmishtha }} \\
=\frac{\frac{0.2}{0.7}+\frac{0.5}{0.8}+\frac{0.8}{0.9}+\frac{1}{1}}{\text { Priti }}+\frac{\frac{0.3}{0.4}+\frac{0.8}{0.5}+\frac{1}{0.6}+\frac{0.4}{0.7}}{\text { Rina }}+\frac{\frac{1}{0}+\frac{0.8}{0.1}+\frac{0.5}{0.2}+\frac{0.2}{0.3}}{\text { Sarmishtha }} .
\end{gathered}
$$

$R$ : a fuzzy relation on $U \times V$ 
TABLE 4. The relation $\mathrm{R}$

\begin{tabular}{|c|c|c|c|}
\hline & Priti & Rina & Sarmishtha \\
\hline Aditi & $\frac{0.2}{0.7}$ & $\frac{0.3}{0.4}+\frac{0.8}{0.5}+\frac{0.5}{0.6}+\frac{0.2}{0.7}$ & 0.0 \\
\hline Bidisha & $\frac{0.2}{0.7}+\frac{0.5}{0.8}+\frac{0.8}{0.9}+\frac{1.0}{1.0}$ & $\frac{0.3}{0.7}$ & 0.0 \\
\hline Camellia & 0.0 & $\frac{0.2}{0.4}$ & $\frac{0.8}{0.1}+\frac{0.5}{0.2}+\frac{0.2}{0.3}$ \\
\hline
\end{tabular}

Let $A^{\prime}$ (highly incredible women) be an observation and represented by a fuzzy set of Type-2 over the set $U$ as

$$
A^{\prime}=\frac{\frac{0.36}{0.4}+\frac{1}{0.5}+\frac{0.25}{0.6}+\frac{0.04}{0.7}}{\text { Aditi }}+\frac{\frac{0.09}{0.7}+\frac{0.36}{0.8}+\frac{0.64}{0.9}+\frac{1.0}{1.0}}{\text { Bidisha }}+\frac{\frac{1.0}{0.1}+\frac{0.64}{0.2}+\frac{0.25}{0.3}+\frac{0.04}{0.4}}{\text { Camellia }} .
$$

Now, we find the cylindrical extension of $A^{\prime}$ and let us set $S$ : the cylindrical extension of $A^{\prime}$, a fuzzy relation on $U \times V$.

TABLE 5. S, the cylindrical extension of $A^{\prime}$

\begin{tabular}{|c|c|c|c|}
\hline & Priti & Rina & Sarmishtha \\
\hline Aditi & $\frac{0.36}{0.4}+\frac{1}{0.5}+\frac{0.25}{0.6}+\frac{0.04}{0.7}$ & $\frac{0.36}{0.4}+\frac{1}{0.5}+\frac{0.25}{0.6}+\frac{0.04}{0.7}$ & $\frac{0.36}{0.4}+\frac{1}{0.5}+\frac{0.25}{0.6}+\frac{0.04}{0.7}$ \\
\hline Bidisha & $\frac{0.09}{0.7}+\frac{0.36}{0.8}+\frac{0.64}{0.9}+\frac{1.0}{1.0}$ & $\frac{0.09}{0.7}+\frac{0.36}{0.8}+\frac{0.64}{0.9}+\frac{1.0}{1.0}$ & $\frac{0.09}{0.7}+\frac{0.36}{0.8}+\frac{0.64}{0.9}+\frac{1.0}{1.0}$ \\
\hline Camellia & $\frac{1.0}{0.1}+\frac{0.64}{0.2}+\frac{0.25}{0.3}+\frac{0.04}{0.4}$ & $\frac{1.0}{0.1}+\frac{0.64}{0.2}+\frac{0.25}{0.3}+\frac{0.04}{0.4}$ & $\frac{1.0}{0.1}+\frac{0.64}{0.2}+\frac{0.25}{0.3}+\frac{0.04}{0.4}$ \\
\hline
\end{tabular}

Next, let $R_{1}=R \circ S=R \sqcap S$ and we obtain 
TABLE 6. The composite relation $R_{1}=R \circ S$

\begin{tabular}{|c|c|c|c|}
\hline & Priti & Rina & Sarmishtha \\
\hline Aditi & $\frac{0.04}{0.7}$ & $\frac{0.3}{0.4}+\frac{0.8}{0.5}+\frac{0.25}{0.6}+\frac{0.04}{0.7}$ & 0.0 \\
\hline Bidisha & $\frac{0.09}{0.7}+\frac{0.36}{0.8}+\frac{0.64}{0.9}+\frac{1.0}{1.0}$ & $\frac{0.09}{0.7}$ & 0.0 \\
\hline Camellia & 0.0 & $\frac{0.04}{0.4}$ & $\frac{0.8}{0.1}+\frac{0.5}{0.2}+\frac{0.2}{0.3}$ \\
\hline
\end{tabular}

Now the projection of $R_{1}$ on $V$ is

$$
B^{\prime}=\frac{\frac{0.09}{0.7}+\frac{0.36}{0.8}+\frac{0.64}{0.9}+\frac{1}{1}}{\text { Priti }}+\frac{\frac{0.3}{0.4}+\frac{0.8}{0.5}+\frac{0.20}{0.6}+\frac{0.09}{0.7}}{\text { Rina }}+\frac{\frac{0.8}{0.1}+\frac{0.5}{0.2}+\frac{0.2}{0.3}}{\text { Sarmishtha }} .
$$

Defuzzification of $B^{\prime}$ is $\frac{1.0}{\text { Priti }}+\frac{0.5}{\text { Rina }}+\frac{0.1}{\text { Sarmishtha }}$.

We conclude that Priti is a very highly smart girl, Rina is a more or less smart girl and Sarmishtha is not at all a smart girl.

Approximate reasoning using the scheme

\section{from ' $\mathrm{X}$ is $A^{*}$ ' and 'if $\mathrm{X}$ is $\mathrm{A}$ then $\mathrm{Y}$ is $\mathrm{B}$ ' infer ' $\mathrm{Y}$ is $B^{*}$ ',}

is termed as the Generalized Modus Ponens. Here, $A, A^{\prime}$ are fuzzy subsets of Type-2 defined over the universe of discourse $U ; B$ and $B^{\prime}$ are these defined over the universe of discourse $V$. Interpreting $(A \Longrightarrow B)$ as a Type-2 binary fuzzy relation $R$,

i.e., $(A \Longrightarrow B)=R(A, B)$ and using Compositional rule of inference, we have deduced $A^{\prime} \circ R=B^{\prime}$. The scheme can be best described in Table 7 .

TABle 7. Generalized Modus Ponens

$$
\begin{aligned}
& \mathrm{p}: \text { if } X \text { is } A \text { then } Y \text { is } B \\
& \begin{array}{ll}
\mathrm{q}: & X \text { is } A^{\prime} \\
\hline \mathrm{r}: & Y \text { is } B^{\prime} .
\end{array}
\end{aligned}
$$

Example 5.2. Consider two Type-2 fuzzy sets $A$ and $B$ as given in Example 8.. Taking $A^{\prime}$, same as $A$, by the equation we have the consequent $B^{\prime}$ as in the following

$$
\begin{aligned}
& B_{v_{1}}^{\prime}=\frac{0}{0}+\frac{0}{.2}+\frac{0}{.4}+\frac{.5}{.6}+\frac{.7}{.8}+\frac{.9}{1} \\
& B_{v_{2}}^{\prime}=\frac{1}{0}+\frac{.8}{.2}+\frac{.5}{.4}+\frac{.4}{.6}+\frac{.1}{.8}+\frac{0}{1} \\
& B_{v_{3}}^{\prime}=\frac{.4}{0}+\frac{.8}{.2}+\frac{1}{.4}+\frac{.8}{.6}+\frac{.5}{.8}+\frac{.2}{1} .
\end{aligned}
$$


and this is a graphical comparison between antecedents and corresponding consequent.

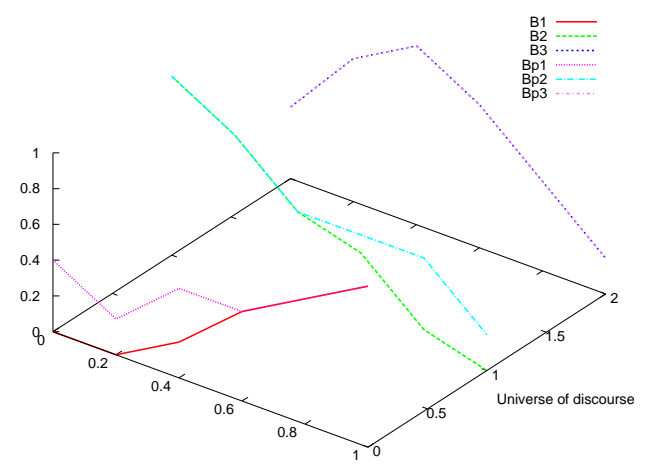

Figure $1 . B$ and $B^{\prime}$

$$
\text { Considering } \begin{aligned}
A^{\prime}=\left\{\frac{A_{u_{1}}^{\prime}}{u_{1}}+\frac{A_{u_{2}}^{\prime}}{u_{2}}+\frac{A_{u_{3}}^{\prime}}{u_{3}}\right\} \text { as } \\
A_{u_{1}}^{\prime}=\frac{1}{0}+\frac{.9}{.2}+\frac{.7}{.4}+\frac{.63}{.6}+\frac{0.32}{.8}+\frac{0}{1}, \\
A_{u_{2}}^{\prime}=\frac{.63}{0}+\frac{.95}{.2}+\frac{1}{.4}+\frac{.95}{.6}+\frac{.7}{.8}+\frac{.45}{1} \\
A_{u_{3}}^{\prime}=\frac{0}{0}+\frac{0}{.2}+\frac{.45}{.4}+\frac{.7}{.6}+\frac{.84}{.8}+\frac{.95}{1} .
\end{aligned}
$$

we have $B=\left\{\frac{B_{v_{1}}}{v_{1}}+\frac{B_{v_{2}}}{v_{2}}+\frac{B_{v_{3}}}{v_{3}}\right\}$ as follows

$$
\begin{aligned}
& B_{v_{1}}^{\prime}=\frac{.23}{0}+\frac{.1}{.2}+\frac{.45}{.4}+\frac{.7}{.6}+\frac{.84}{.8}+\frac{.95}{1} \\
& B_{v_{2}}^{\prime}=\frac{1}{0}+\frac{.9}{.2}+\frac{.7}{.4}+\frac{.63}{.6}+\frac{.32}{.8}+\frac{.25}{1} \\
& B_{v_{3}}^{\prime}=\frac{.63}{0}+\frac{.9}{.2}+\frac{1}{.4}+\frac{.9}{.6}+\frac{.7}{.8}+\frac{.45}{1}
\end{aligned}
$$

and a graphical comparison between antecedents and corresponding consequent is as given in the following Figure 2. and Figure 3. respectively. 


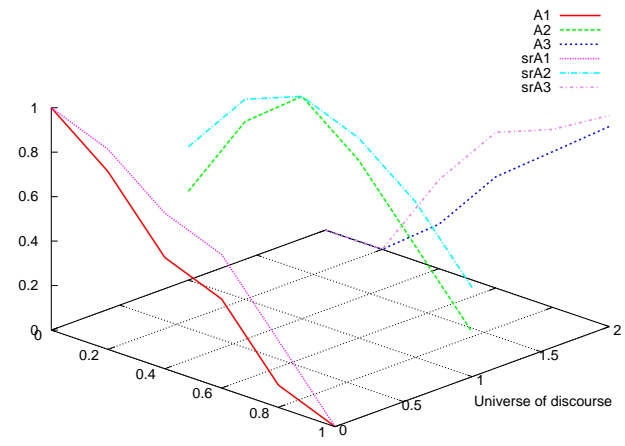

Figure 2. $A$ and $\sqrt{A}$

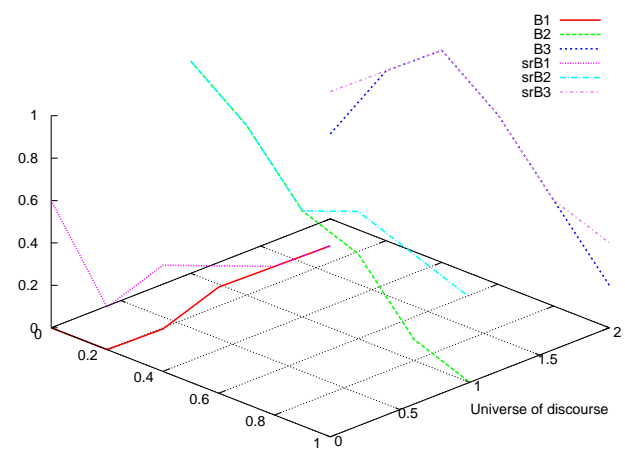

Figure $3 . \quad B$ and $\sqrt{B}$

and

$$
\begin{aligned}
& A_{u_{1}}^{\prime}=\frac{1}{0}+\frac{.64}{.2}+\frac{.25}{.4}+\frac{.16}{.6}+\frac{.01}{.8}+\frac{0}{1}, \\
& A_{u_{2}}^{\prime}=\frac{.16}{0}+\frac{.64}{.2}+\frac{1}{.4}+\frac{.64}{.6}+\frac{.25}{.8}+\frac{.04}{1}, \\
& A_{u_{3}}^{\prime}=\frac{0}{0}+\frac{0}{.2}+\frac{.04}{.4}+\frac{.25}{.6}+\frac{.49}{.8}+\frac{.81}{1},
\end{aligned}
$$


as $A^{\prime}=\left\{\frac{A_{u_{1}}^{\prime}}{u_{1}}+\frac{A_{u_{2}}^{\prime}}{u_{2}}+\frac{A_{u_{3}}^{\prime}}{u_{3}}\right\}$ gives $B=\left\{\frac{B_{v_{1}}}{v_{1}}+\frac{B_{v_{2}}}{v_{2}}+\frac{B_{v_{3}}}{v_{3}}\right\}$ as

$$
\begin{aligned}
& B_{v_{1}}^{\prime}=\frac{0}{0}+\frac{0}{.2}+\frac{.2}{.4}+\frac{.34}{.6}+\frac{.49}{.8}+\frac{.81}{1}, \\
& B_{v_{2}}^{\prime}=\frac{1}{0}+\frac{.64}{.2}+\frac{.5}{.4}+\frac{.24}{.6}+\frac{.01}{.8}+\frac{0}{1}, \\
& B_{v_{3}}^{\prime}=\frac{.4}{0}+\frac{.64}{.2}+\frac{1}{.4}+\frac{.64}{.6}+\frac{.29}{.8}+\frac{.11}{1} .
\end{aligned}
$$

and a graphical comparison between antecedents and corresponding consequent is given in Figure 4. and Figure 5 .respectively.

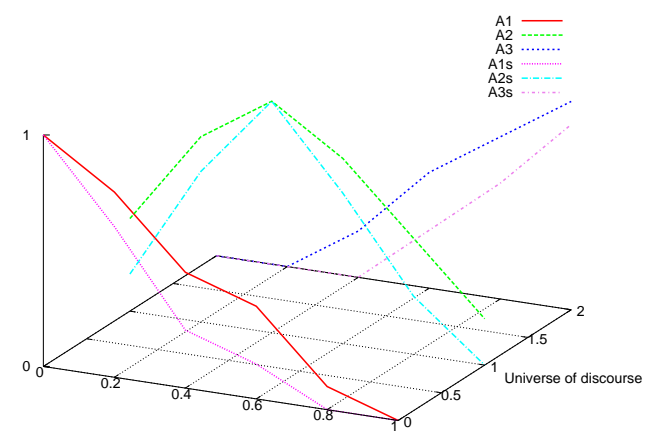

Figure $4 . A$ and $A^{2}$

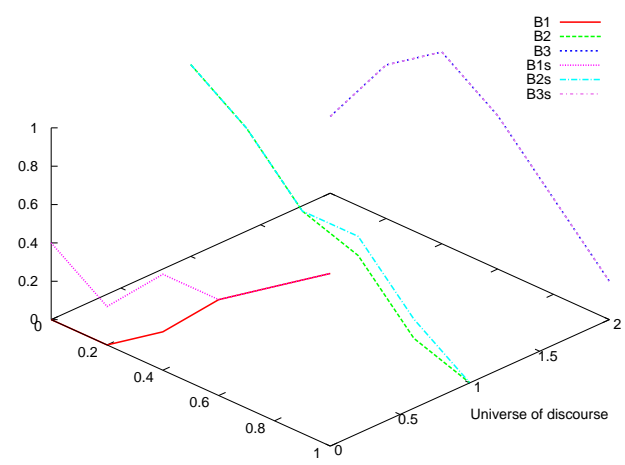

Figure $5 . B$ and $B^{2}$ 
ALGORITHM RBAR : Rule-based Approximate Reasoning (GMP)

\author{
Input : $A^{\prime}$ and $A \rightarrow B$ \\ Output : $B^{\prime}$
}

Step 1. Represent $A, A^{\prime}$ as Type-2 fuzzy subsets of $U$ and $B$ as a Type-2 fuzzy subset of $V$.

Step 2. Compute $I$ as a Type-2 fuzzy relation over the universe of discourse $U \times V$. Step 3. Compose $A^{\prime}$ and $I$ to obtain $B^{\prime}$ using some conjunction operation meant for fuzzy sets of Type-2, as in the following.

Let $A$ and $B$ be defined as earlier then

$$
I=\sum_{(x, y) \in X \times Y} \frac{I(x, y)=\sum_{j \in[0,1]} \frac{I_{(x, y)}(j)}{j}}{(x, y)} .
$$

If now,

$$
A^{\prime}=\sum_{x \in X} \frac{A^{\prime}(x)=\sum_{j \in[0,1]} \frac{A_{x}^{\prime}(j)}{j}}{x}
$$

then

$$
B^{\prime}=A^{\prime} \circ I=\sum_{y \in Y} \frac{B^{\prime}(y)}{y}
$$

where,

$$
B^{\prime}(y)=\sup _{x \in X}\left(A^{\prime}(x) * I(x, y)\right),
$$

(here $A^{\prime}(x)$ and $I(x, y)$ both identify fuzzy subsets of $[0,1]$ )

$$
\text { i.e., } B^{\prime}(y)=\sup _{x \in X}\left\{\left(A^{\prime}(x) * I(x, y)\right)\right\} \text {, }
$$

where $*$ is taken as the conjunction operation from the lattice $F$.

\title{
6. CONCLUDING REMARKS
}

Any attempt to study Type-2 fuzzy logic and the corresponding theory of Type-2 fuzzy sets is still considered to be difficult. Moreover, any study on the possibility of using Type-2 fuzzy logic in handling uncertainties in rule-based systems is interesting and important. It has already been established that approximate reasoning is an important topic of research because of its scope of applications in different fields of research particularly, in fuzzy control. This research on modelling approximate reasoning using Type-2 fuzzy set theory will definitely help the research community. It is hoped that with modelling of Generalized Modus Ponens and Compositional Rule of Inference using Type-2 fuzzy set theory, approximate reasoning methodology can be made more versatile in so far as decision-making under uncertainty is concerned. 


\section{Acknowledgement.}

Phase-III of University Grants Commission funded Special Assistance Programme has provided necessary financial support to this work conducted at the Department of Mathematics, Visva-Bharati. It is also supported by the UGC fellowship (Serial no. 2121540996 and Roll no. 423697 under the UGC scheme). UGC's financial support is highly appreciated.

\section{REFERENCES}

[1] Galatos N., Residuated lattices: An algebraic glimpse at substructural logics, Elsevier, 2007.

[2] Hajek P., Metamathematics of fuzzy logic, Kluwer Academic Press, The Netherlands, 1998.

[3] John R.I., Type-2 fuzzy sets: an appraisal of theory and applications, Internat. J. Uncertainty, Fuzziness and Knowledge-Based Systems, 6(6), (1998), 563-576.

[4] Liang Q., Karnik N.N., and Mendel J.M., Type-2 fuzzy logic systems, IEEE Transactions on Fuzzy Systems, 7(6), (1999), 643-658.

[5] Mendel J. and John R., Type-2 fuzzy sets made simple, IEEE Transactions on Fuzzy Systems, 10(2), (2002), 117-127.

[6] Mizumoto M. and Kokichi T., Fuzzy-Fuzzy Automata, Kybernetes, 5, (1976), 107-112.

[7] Mizumoto M. and Kokichi T., Some properties of fuzzy sets of Type 2, Information and Control, 31, (1976), 312-340.

[8] Mizumoto M. and Kokichi T., Fuzzy Sets of Type-2 under Algebraic Product and Algebraic Sum, Fuzzy Sets and System, 5, (1981), 277-290.

[9] Morgan W. and Dilworth R.P., Residuated lattices, Transactions on American Mathematical Society, 45, (1939), 335-354.

[10] Pavelka J.P., On fuzzy logic Part I, Zeistchr. f. math. Logik and Grundlagun d. Math. Bd, 25, (1979), 45-52.

[11] Pavelka J.P., On fuzzy logic Part II, Zeistchr. f. math. Logik and Grundlagun d. Math. Bd, 25, (1979), 119-134.

[12] Pavelka J.P., On fuzzy logic Part III, Zeistchr. f. math. Logik and Grundlagun d. Math. Bd, $25,(1979), 447-464$.

[13] Walker C.L. and Walker E.A., The algebra of fuzzy truth values, International Journal of Approximate Reasoning, 149, 2005, pp. 309-347.

[14] Walker C.L. and Walker E.A., Automorphisms of the algebra of fuzzy truth values, Internat. J. Uncertainty, Fuzziness and Knowledge-Based Systems, 14, (2006), 711-732.

[15] Walker C.L. and Walker E.A., Sets with type-II operations, Internat. J. Approximate Reasoning, 50, (2009), 63-71.

[16] Zadeh L.A., Fuzzy sets, Information and Control, 8, (1965), 338-353.

[17] Zadeh L.A., The concept of linguistic variable and its application to approximate reasoning I, Information Sciences, 8, (1975), 199-249.

[18] Zadeh L.A., A computational approach to fuzzy quantifires in natural languages, Computers and Mathematics with applications, 9, (1983), 149-184. 